\title{
Sex effect on presenilins expression in post-natal rat brain
}

\author{
Paola Piscopo $^{1 *}$, Sonia Canterini ${ }^{2 *}$, Valentina Carletti ${ }^{1,2}$, Paolo Rosa ${ }^{1}$, Alessio Crestini ${ }^{1}$, \\ Maria Teresa Fiorenza ${ }^{2}$, Annamaria Confaloni ${ }^{1 \#}$ \\ ${ }^{1}$ Department of Cellular Biology and Neuroscience, Section of Clinics, Diagnosis and Therapy of Central Nervous System Diseases, \\ Istituto Superiore di Sanità, Rome, Italy \\ ${ }^{2}$ Department of Psychology, Section of Neuroscience, "Sapienza" University of Rome, Rome, Italy \\ Email: " annamaria.confaloni@iss.it
}

Received 8 August 2013; revised 12 November 2013; accepted 28 November 2013

Copyright (C) 2013 Paola Piscopo et al. This is an open access article distributed under the Creative Commons Attribution License, which permits unrestricted use, distribution, and reproduction in any medium, provided the original work is properly cited.

\begin{abstract}
Presenilin 1 and presenilin 2 are widely expressed during brain development. Several mutations in these proteins have been associated with autosomal-dominant inherited forms of Alzheimer disease. Their expression is regulated by various cellular and extracellular factors, which change with age and sex. Both age and sex are key risk factors for Alzheimer's disease, but the issue of whether the expression of presenilins is influenced by the sex during early postnatal development of the brain has been poorly investigated so far. In this study, we report that transcript levels of presenilins, and the subset of neurons expressing these proteins in various brain areas of the developing post-natal brain are different in male and female rats, suggesting that their function(s) may contribute to sexual dimorphism in the brain, both at morphological and functional levels.
\end{abstract}

Keywords: Development; Sex; Presenilins; $\gamma$-Secretase; Rats; Sexual Dimorphism

\section{INTRODUCTION}

Presenilin 1 (PSEN1), along with its homologue Presenilin 2 (PSEN2), constitutes the catalytic core of the $\gamma$-secretase complex: three additional constituents of this complex include the subunits APH1 (anterior pharynx defective 1), PEN2 (presenilin enhancer 2), and NCSTN (nicastrin) [1]. PSEN-dependent cleavage of the amyloid- $\beta$ (A $\beta$ ) precursor protein (APP) contributes to the generation of $\mathrm{A} \beta$ peptides, and to the formation of the pathological $\mathrm{A} \beta$ senile plaques. Several mutations in the genes of presenilins (PSENs) modify the $\gamma$-secretase cleavage of APP, resulting in an altered ratio of $\mathrm{A} \beta 42 / \mathrm{A} \beta 40$;

${ }^{*}$ These authors contributed equally to the study.

${ }^{\#}$ Corresponding author. these gene mutations are considered to be the major cause of Familial Alzheimer Disease (FAD) [2]. The role of $\gamma$-secretase in $\mathrm{A} \beta$ production and in the pathogenesis of Alzheimer's disease (AD) is well established. However, recent reports suggest that co-factors of the enzymatic complex have biological functions that contribute to other non-disease related pathways. Moreover, $\gamma$-secretase has important roles in embryonic development through the regulation of specific signal transduction pathways [3]. Strong expression of PSENs has been observed in the cells of the ventricular zone of early and late rat embryos followed by a gradual decrease during post-natal development [4]. Song and colleagues proposed that during early embryogenesis the role of PSENs is most probably related to Notch cleavage [5]. Loss of PSEN1 expression in mice results in lethality at early stages of development [6], with embryos having characteristics similar to those of Notch-null mice [7]. The relative levels of PSEN1/PSEN2 mRNAs vary among different tissues at different stages of brain development: PSEN1 mRNA levels are approximately three-fold higher than those of PSEN2 in the brains of developing humans and mice, suggesting that the expression of PSEN transcripts is differentially regulated during nervous system maturation $[8,7]$. PSEN1 expression peaks at post-natal day 10 (P10), particularly in the cerebellum and hippocampus [9]. A recent work by Kumar and Thakur reported that PSEN1 expression is upregulated from $\mathrm{P} 0$ to $\mathrm{P} 45$ in mice, a critical time window for synaptogenesis, including the formation of new synapses and the elongation of axons and dendrites, while PSEN2 mRNA showed no significant change [10]. These developmentally regulated differences in temporal-spatial expression of PSENs are likely due to presence of large numbers of extracellular signaling molecules such as neurotrophins and neuromodulators that act through distinct pathways to regulate PSENs expression 
$[11,12]$. The activity of PSENs is essential for synaptic function, memory formation, and neuronal survival during the whole span of life. Furthermore, their role in normal and pathological aging has been extensively confirmed. Recent reports have shed light on modulation of PSENs in adult and aged animal models with the aim to better understand their regulatory pathways. Ghosh and Thakur document that presenilin expression in the cerebral cortex of aged mice is regulated by sex steroids $[13,14]$. Furthermore, Bowen and colleagues showed that in cases of post-menopause/andropause, increased level of luteinizing hormone alters the processing of APP towards the amyloidogenic pathway [15]; however, this alteration is not mediated via enhanced $\gamma$-secretase activity or PSENs expression. Such changes may affect the functional integrity of the multimeric $\gamma$-secretase complex and other related functions. Expression of genderspecific differences associated with the risk of a disorder can provide clues about its pathogenesis. In $\mathrm{AD}$, gender is a risk factor associated with the sporadic form of dementia. In this regard, Placanica and colleagues report an increase of $\gamma$-secretase activity in aged females that may contribute to the increased incidence of sporadic $A D$ in women, which might explain the aggressive $\mathrm{A} \beta$ plaque pathology seen in female mouse models of $\mathrm{AD}[16,17]$. Several authors investigated the effect of the sex on aging brain, with particular reference to the expression and activity of components of the $\gamma$-secretase complex $[14,16,18]$; however, the effect of the sex on patterns of presenilin expression during early post-natal development of the brain has been mostly neglected so far. By addressing this issue in developing post-natal brain of rats, we found that sex affects transcriptional expression of PSENs, and also the subset of neurons in which these proteins are observed, suggesting that PSENs may contribute to sexual dimorphism in the brain, both at the morphological and functional levels.

\section{MATERIALS AND METHODS}

\subsection{Animals and Sample Collections}

Wistar rats were purchased from Charles River Italia (Calco, Italy) and housed in temperature-and humiditycontrolled quarters $\left(21^{\circ} \mathrm{C}+/-1{ }^{\circ} \mathrm{C} ; 60 \%+/-10 \%\right.$ relative humidity), with a white/red light cycle (white light on from 8:30 am to $2: 30 \mathrm{pm})$. Rats of both sexes were used at P0 (day of birth), P7, P14 and adult (225 - $275 \mathrm{~g}$ ). The experimental protocol was carried out according to the EC guidelines [19] (EC Council Directives 86/609 1987), and the Italian legislation, under permission of the Italian Ministry of Health. Moreover, all efforts were made to minimize the number of animals used and their suffering. For quantitative analysis, rats were quickly decapitated, their brains rapidly removed and placed on an iced plate, then dissected under a binocular microscope. Frontoparietal cortex was dissected out bilaterally, the hippocampus excised at the level of the midbrain, and the cerebellum was removed. All tissues were immediately dipped in RNA Later (Qiagen) and stored at $-80^{\circ} \mathrm{C}$ until used. For immunohistochemistry, pups and post-natal rats of both sexes (P0 through adulthood), were anesthetized with intraperitoneal sodium pentobarbital, and perfused through the heart with a wash solution of $0.9 \mathrm{M}$ phosphate buffer, followed by a fixative consisting of $4 \%$ paraformaldehyde.

\subsection{RNA Extraction and Quantitative Real-Time PCR}

RNA was collected as previously described [20]. Briefly, total RNA from $10 \mathrm{mg}$ rat tissue was extracted (RNeasy Lipid Tissue, Qiagen) according to the manufacture's instructions, and was stored at $-80^{\circ} \mathrm{C}$, until used. cDNAs were synthesized from $2 \mu \mathrm{g}$ of total RNA of each sample, with use of Superscript II reverse transcriptase and random primers (Invitrogen Inc., Carlsbad, CA) according to the manufacturer's instructions. Each cDNA synthesis reaction was performed with specific primers and probes (Applied Biosystems). Reverse transcription quantitative real-time PCR (RT-qPCR) was carried out using ABI PRISM 7000 sequencer. A $20 \mu 1$ reaction mixture containing $2 \mu \mathrm{l}$ of cDNA template, $8 \mu \mathrm{l}$ TaqMan Universal PCR Master Mix and $1 \mu \mathrm{l}$ primer probe mixture was amplified, using the following parameters: denaturation at $95^{\circ} \mathrm{C}$ for $2 \mathrm{~min}, 40$ cycles of $95^{\circ} \mathrm{C}$ for $20 \mathrm{sec}$ and $60^{\circ} \mathrm{C}$ for $45 \mathrm{sec}$. Relative expression of mRNA was calculated using the comparative $\mathrm{Cq}$ method and the $18 \mathrm{~S}$ gene as an endogenous reference.

\subsection{Statistical Analysis}

All experiments were repeated in triplicate, with samples obtained from different animals $(\mathrm{n}=4)$. Statistical analysis was performed using the t-test analysis for comparing two selected groups having one variable. $p$ values $<0.05$ were considered significant $(95 \%$ confidence interval). The data from the developing rat brains are presented as mean \pm S.E.M.

\subsection{Immunohistochemistry}

For immunohistochemistry, perfused brains were quickly dissected, post-fixed in $4 \%$ PFA at $4^{\circ} \mathrm{C}$, dehydrated, embedded in Paraplast Plus Tissue Embedding Medium (Società Italiana Chimici, Roma, Italy), and then serially sectioned (slice thickness, 9 um). Slices were mounted on Superfrost Exell adhesion slides (Thermo Scientific, Milan, Italy), de-waxed in xylene, rehydrated, and washed in PBS. Following antigen unmasking in $10 \mathrm{mM}$ sodium citrate buffer at $\mathrm{pH} 6.0$ and post-fixation with $4 \%$ 
PFA for $10 \mathrm{~min}$, non-specific binding was blocked with $5 \%$ bovine serum albumin and $0.2 \mathrm{M}$ glycine in PBS for $3 \mathrm{~h}$ at room temperature (RT). Endogenous peroxidases were inactivated by treatment with $0.3 \% \mathrm{H}_{2} \mathrm{O}_{2}$ for $15 \mathrm{~min}$ at RT. Slides were incubated with rabbit polyclonal antiPSEN1 antibody (Abcam, Cambridge, UK; 1:50 dilution) or with rabbit polyclonal anti-PSEN2 antibody (Santa Cruz, Heidelberg, Germany; 1:50 dilution) at $0^{\circ} \mathrm{C}$ $-4^{\circ} \mathrm{C}$ overnight. Antibody binding was visualized using biotinylated secondary antibodies, followed by processing with the $\mathrm{ABC}$ Elite Kit (Vector laboratories) using DAB-nickel as chromogen. Representative panels of at least 3 experiments are shown in each figure. To assess immunostaing specificity, primary antibodies were omitted in control slices processed in parallel.

\section{RESULTS}

\subsection{Transcriptional Expression of Presenilins Is Developmentally Regulated in the Rat Brain}

PSEN1 and PSEN2 are widely expressed during brain development. Here, we measured the mRNA expression of PSEN1 and PSEN2 in cerebral cortex, hippocampus, and cerebellum at increasing developmental ages (P0, P7, $\mathrm{P} 14$, and adult; $\mathrm{n}=4$ for each time point). Samples were normalized by $18 \mathrm{~S}$ rRNA expression levels. PSEN1 and PSEN2 were expressed in all three brain regions at the four time points evaluated. Quantitative analysis showed that in rat brain of both male and female the expression of both PSENs is gradually reduced in cerebral cortex, hippocampus and cerebellum during brain maturation
(Figures 1 and 2).

\section{Sex-Related Differences in the Expression Level of Presenilins}

To investigate the effect of the sex on the expression of presenilin transcripts, we carried out a quantitative analysis by real time PCR on the cerebral cortex, hippocampus and cerebellum of post-natal and adult male and female rats. Our results showed that female rats display higher levels of PSEN1 transcripts in all three brain regions compared to male rats (Figure 1). In particular, greater differences were observed at $\mathrm{P} 0, \mathrm{P} 7$ and $\mathrm{P} 14$ in the cerebral cortex (P0: male $4.86 \pm 0.73$ vs female $13.36 \pm 1.25 \mathrm{p}<0.05$; P7: male $3.51 \pm 0.32$ vs female $7.07 \pm 0.66 \mathrm{p}<0.05$; P14: male $1.32 \pm 0.14$ vs female $4.37 \pm 0.13 \mathrm{p}<0.01)$ and in the hippocampus at $\mathrm{P} 0$ and P7 (P0: male $6.03 \pm 1.43$ vs female $10.63 \pm 1.37$ $\mathrm{p}<0.05$; P7: male $2.45 \pm 0.45$ vs female $5.02 \pm 0.69$ ), while any specific variation in gene expression attributable to sex were observed in the adult rats. In the cerebellum, PSEN1 expression was similar between male and female at $\mathrm{P} 0$, while at $\mathrm{P} 7$, the expression appeared sex-specific displaying a trend similar to that observed in the cerebral cortex and hippocampus at P7 (male $2.11 \pm$ 0.4 vs female $6.1 \pm 1.23 \mathrm{p}=0.09$ ) and at P14 (male $0.7 \pm$ 0.96 vs female $3.2 \pm 0.7 \mathrm{p}=0.07$ ). As for PSEN2 gene, transcript levels were similar between the sexes (Figure 2 ); the only highly significant sex-dependent variation was found in the cerebellum at P7 (male $1.68 \pm 0.20$ vs female $4.79 \pm 0.17 \mathrm{p}<0.01$ ). These findings prompted us to investigate whether the levels of other $\gamma$-secretase

\section{Presenilin 1}

Cerebral cortex

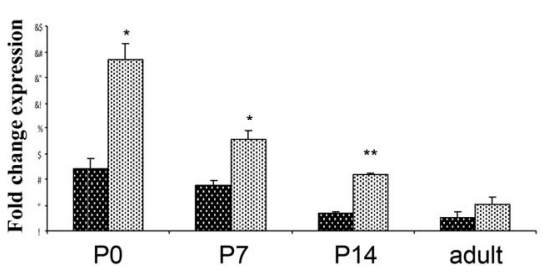

Hippocampus

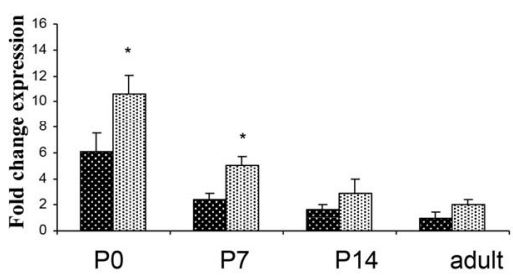

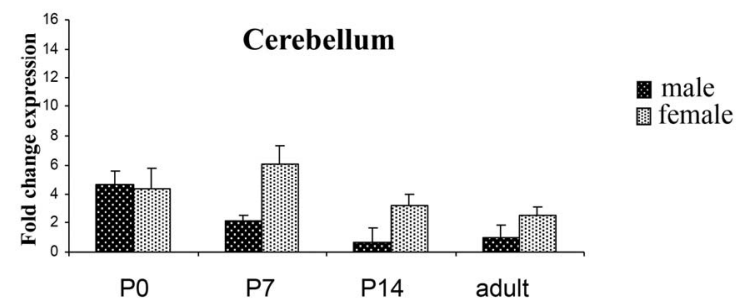

Figure 1. PSEN1 expression in different rat brain areas during post-natal development. Rat PSEN1 expression levels were analyzed by RT-qPCR. Results are represented as normalized mean $( \pm \mathrm{SE})$. Each sample was run both in four biological and three technical replicates. The expression analysis was performed on cerebral cortex, hippocampus and cerebellum at $\mathrm{P} 0, \mathrm{P} 7, \mathrm{P} 14$ and adult. ${ }^{*} \mathrm{p}<0.05 ;{ }^{* *} \mathrm{p}<0.01$. 


\section{Presenilin 2}

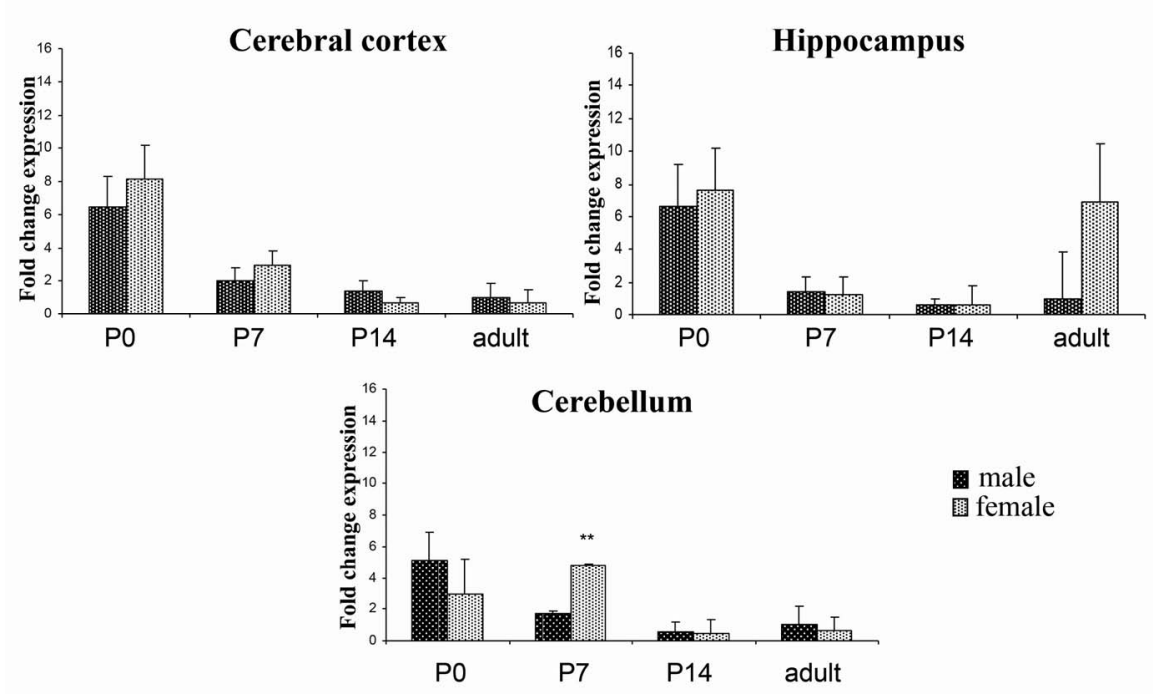

Figure 2. PSEN2 expression in different rat brain areas during post-natal development. Rat PSEN2 expression levels were analyzed by RT-qPCR. Results are represented as normalized mean $( \pm \mathrm{SE})$. Each sample was run both in four biological and three technical replicates. The expression analysis was performed on samples from cortex, hippocampus and cerebellum at P0, P7, P14 and adult. ${ }^{* *} \mathrm{p}<0.01$

protein subunits, namely, nicastrin, APH1a, and PEN2, were also modulated by sex during post-natal development of the brain. However, no significant changes were detected for transcript levels of these genes in any of the brain region analysed (data not shown).

\subsection{Sex-Related Expression of Presenilins in Specific Subsets of Neurons}

To investigate whether the differences in transcripts were detectable also at the protein level and to determine whether PSENs localization varied depending on sex and developmental time, histological sections of the cerebral cortex, hippocampus and cerebellum of male and female rats were analysed by immunohistochemistry with antiPSEN1 or PSEN2 antibodies at increasing post-natal ages (P0, P7, P14 and adult).

\subsubsection{PSEN1 and PSEN2 in the Hippocampus}

In the post-natal hippocampus, neurons expressing PSEN1 and PSEN2 proteins gradually appeared during ontogenesis. Pyramidal neurons in all hippocampal CA sub-fields, and granule cells in the dentate gyrus, were immunostained with both antibodies. More specifically, males and females showed similar patterns and intensities of immunostaining at $\mathrm{P} 0$ and at P7 (Figure 3); however, at P14 females displayed higher levels of both PSENs compared to males. Immunostaining specificity was assessed in parallel processed control slices, in which primary antibodies were omitted (control panel, CTRL). Hippocampus of adult brain displayed a pattern of PSEN1 and PSEN2 expression similar to that of P14 (data not shown).

\subsubsection{PSEN1 and PSEN2 in the Frontoparietal Cortex}

At P0, immunostaining for PSEN1 and PSEN2 in adjacent sections appeared robust; the proteins were ubiquitously distributed throughout the six layers of the cerebral cortex, in both males and females. However at P7 and P14, sex-specific differences in PSENs expression were readily observed: males showed maximal PSEN1 and PSEN2 immunostaining in a population of neurons corresponding to the intermediate zone of the developing cerebral cortex (layers II/III), with scattered cells residing in IV layer, but never in the pyramidal cell layers (layers V and VI). In contrast, females displayed a more homogenous expression of both PSENs throughout all cortical layers, including layer $\mathrm{V}$ in which pyramidal cells displayed high levels of both PSEN1 and PSEN2 (Figure 4). Immunostaining specificity was assessed in parallel processed control slices, in which the primary antibodies were omitted. Frontoparietal cortex of adult brain displayed a pattern of PSEN1 and PSEN2 expression similar to that of P14 (data not shown).

\subsubsection{PSEN1 and PSEN2 in the Cerebellum}

In the post-natal cerebellum, immunoreactivity for PSEN1 was detected throughout the whole developing cerebral cortex (external to internal granule layer, EGL and IGL) of both males and females at P0 and P7; 


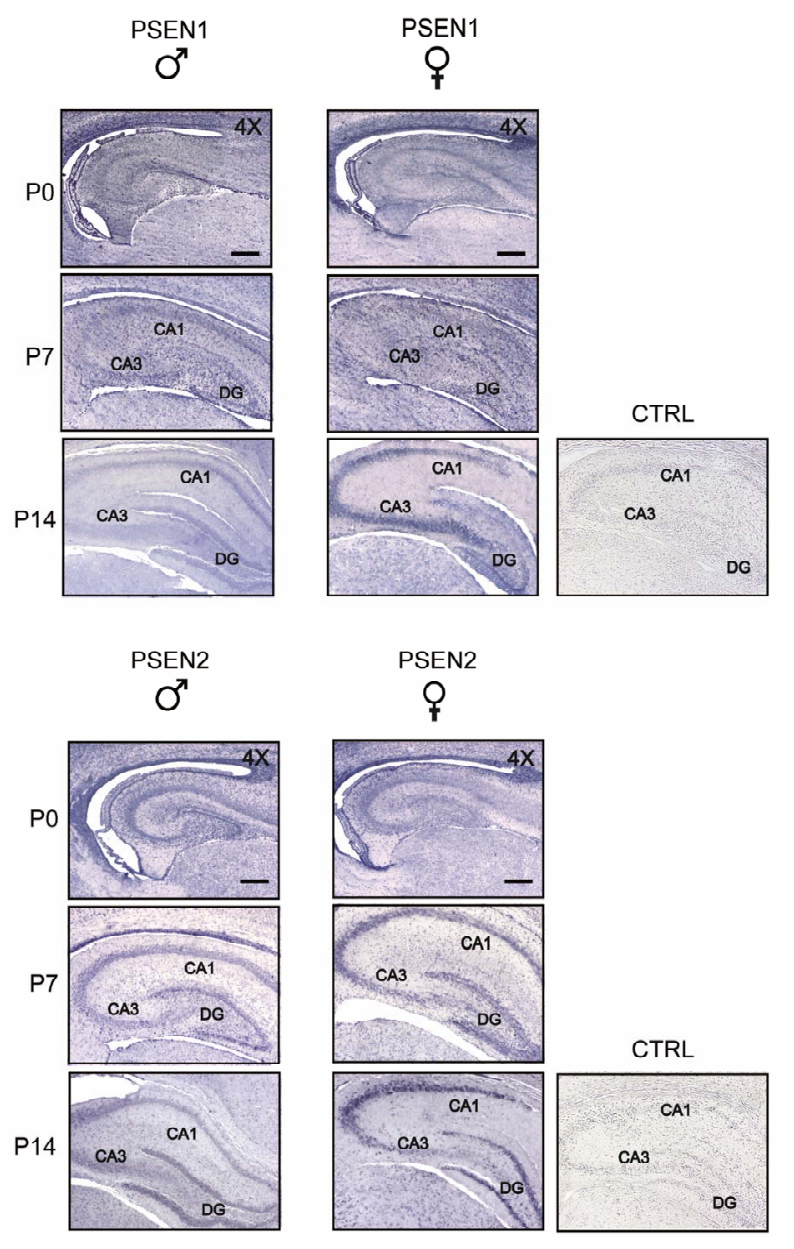

Figure 3. Comparison of PSEN1 and PSEN2 expression in post-natal developing hippocampus of male and female rats. Coronal sections were obtained from rats at increasing postnatal ages, days $0,7,14(\mathrm{P} 0-14)$ and processed by immunohistochemistry using anti-PSEN1 and anti-PSEN2 antibodies. PSEN1 and PSEN2 immunostaining gradually appears during ontogenesis staining pyramidal neurons in all hippocampal CA sub-fields (CA1-CA3) and granule cells in the dentate gyrus (DG) of both males and females. CTRL, representative panel showing a control slice in which the primary antibody was omitted. Scale bar $=350 \mu \mathrm{m}$

however at P14, PSEN1 immunoreactivity was visible in the EGL and IGL of male rats, whereas it was absent in the female EGL and barely detectable in the female IGL. At this stage, PSEN1 expression was a hallmark of Purkinje cell somata in both males and females, with the exclusion of most part of the nuclear compartment except nucleoli. As for PSEN2 expression, our immunohistochemical analyses showed a temporal and spatial pattern similar to that of PSEN1, confirming the existence of sex-dependent differences particularly evident in the EGL and IGL of P14 rats. Compared to PSEN1 expression, however, the expression of PSEN2 in Purkinje cells appeared earlier and clearly detectable at P7 in both males and females (Figure 5). Immuno-

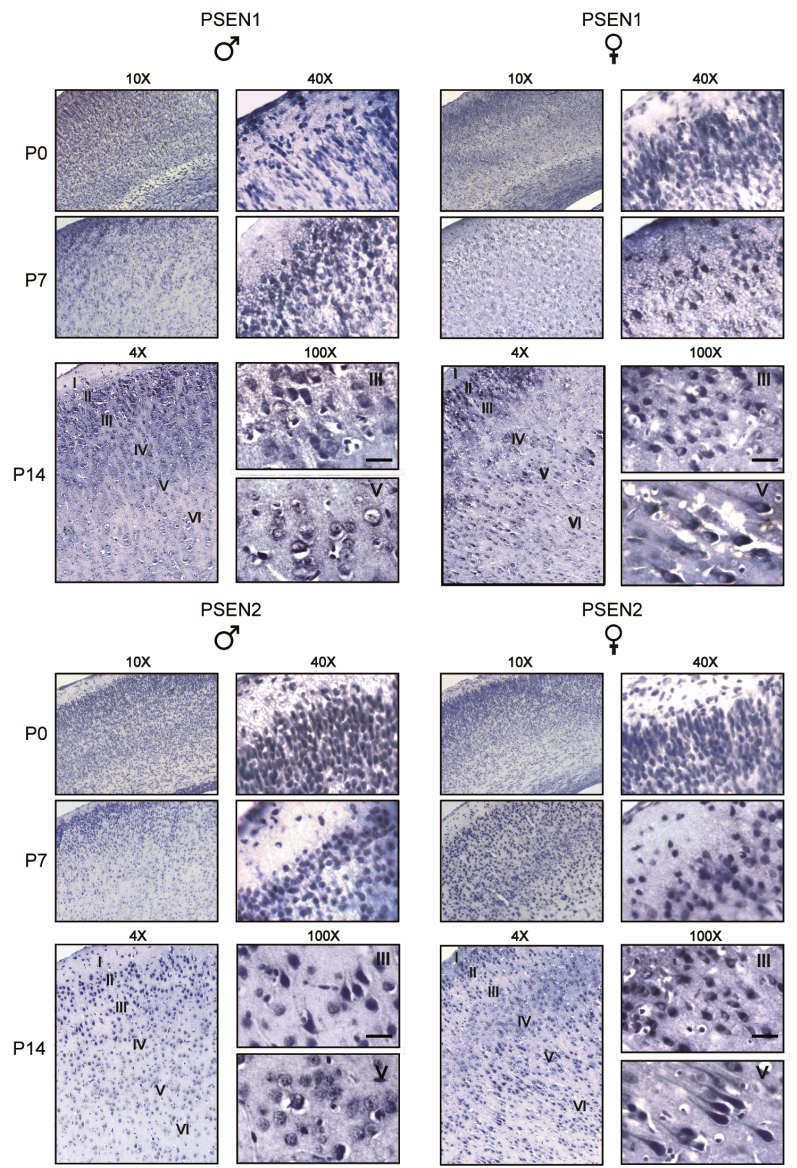

Figure 4. Comparison of PSEN1 and PSEN2 expression in post-natal developing frontotemporal cortex of male and female rats. Coronal sections were obtained from rats at increasing postnatal ages, days $0,7,14$ (P0-14) and processed by immunohistochemistry using anti-PSEN1 and anti-PSEN2 antibodies. Note that PSEN1 and PSEN2 immunostaining appears ubiquitously distribued throughout the six cortex layers at P0 and P7 in both males and females. However, starting from P7, females display a more robust expression of both PSEN1 and PSEN2 in pyramidal cells of layers V and VI. Cortical layers (I-VI) are indicated. Scale bar $=50 \mu \mathrm{m}$.

staining specificity was assessed in parallel processed control slices, in which the primary antibodies were omitted. Cerebellum of adult brain displayed a pattern of PSEN1 and PSEN2 expression similar to that of P14 (data not shown).

\section{DISCUSSION}

The importance of presenilins in $\mathrm{AD}$ is highlighted by the almost 200 mutations in PSEN1 and the 20 mutations in PSEN2 genes that have been associated with this disease, all of which are causative for autosomal-dominant inherited AD [21,22]. Presenilin expression is regulated by several cellular and extracellular factors, which change with age and sex. Both age and sex are key risk factors for Alzheimer's disease. In fact, a number of 


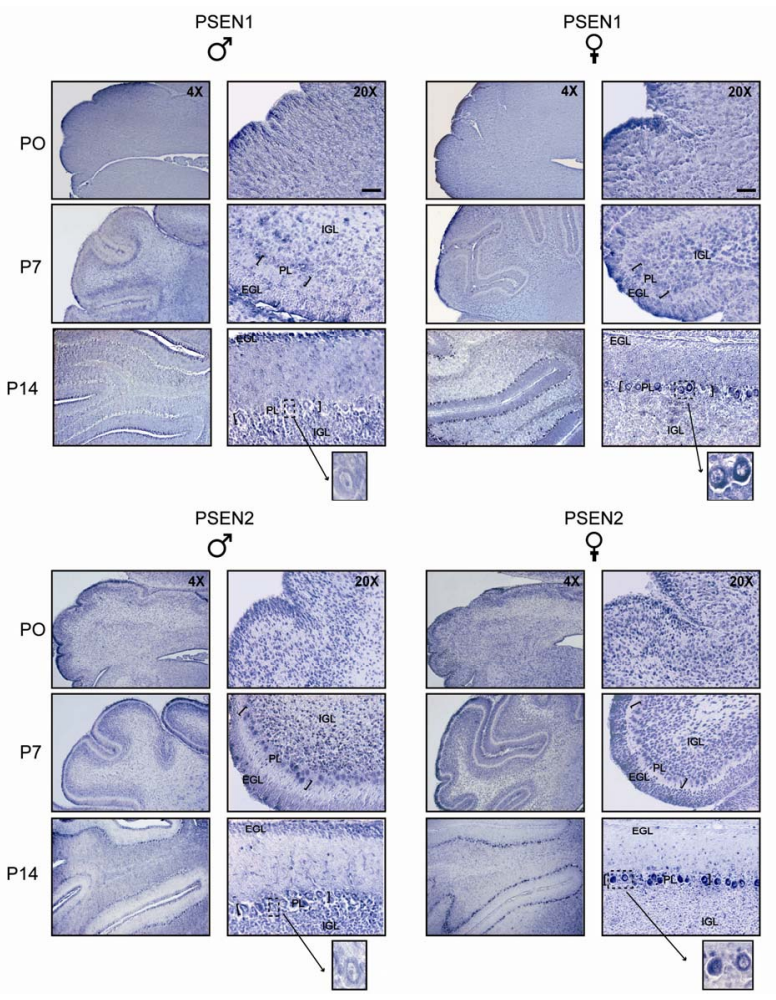

Figure 5. Comparison of PSEN1 and PSEN2 expression in post-natal developing cerebellum of male and female rats. Coronal sections were obtained from rats at increasing postnatal ages, days $0,7,14$ (P0-14) and processed by immunohistochemistry using anti-PSEN1 and anti-PSEN2 antibodies. At P0 and P7, both males and females express PSEN1 and PSEN2 in the external granular layer (EGL) and internal granular layer (IGL). At P14, PSEN1 and PSEN2 immunoreactivity is still detected in males EGL and IGL, whereas it is barely detected in the females ones. By contrast, at this stage, females express high level of both PSEN1 and PSEN2 in Purkinje cells somata. Inserts represent higher magnification $(100 \times)$ of the corresponding photomicrographs, showing stained Purkinje cell $(\mathrm{PC})$ bodies. Scale bar $=120 \mu \mathrm{m}$.

studies have shown that women have a higher prevalence and risk for $\mathrm{AD}[23,24]$. At a molecular level, Placanica and colleagues suggested that the increased $\gamma$-secretase activity observed in aged females may contribute to the increased incidence of sporadic AD reported in women, in addition to the aggressive A-beta plaque pathology seen in female mouse models of AD [16]. These reports led several authors to investigate the effect of the sex on aging brain, with particular reference to the expression and the activity of components of the $\gamma$-secretase complex. Other authors showed that cortical expression of APP mRNA is lower in female mice as compared to males, and is regulated by sex steroids. Interestingly, these findings are in agreement with the higher level of methylation detected on the APP promoter in females [25]. More recently Ghosh and Thakur demonstrated that gonadal steroids are able to modulate the presenilins expression in the cerebral cortex of aged mice $[13,14,18]$ and that the androgen depletion leads to an higher PSEN1 expression in the male hippocampus [26]. However, whether or not presenilin expression is directly affected by sex-specific factors during early post-natal development remains unknown. The possibility of a direct influence is suggested by the findings that neural sexual dimorphism is established during late embryonic and early post-natal development, concomitantly with the increase of testosterone production by the neonatal testes. Testosterone regulates transcription via androgen receptors or, after it is aromatized to estradiol, via estrogen receptors [27]. These receptors in turn influence gene expression by acting in complexes with co-activators and co-repressors, several of which have enzymatic activities modifying histones status [28]. Interestingly, both histone-acetylation and methylation are sexually dimorphic in the developing cortex and hippocampus of the mouse during the first post-natal week [29].

Our data also suggest the existence of a dimorphic expression of presenilin transcripts, which is particularly marked in the cerebral cortex and hippocampus of rats. In fact, the PSEN1 expression profile in the cerebral cortex is significantly different in females compared to males during post-natal development. We observed an increase of at least 2.0 fold in females vs males for PSEN1 gene expression at P7 and of more than 3.0 fold by P14. These different expression levels in the early post-natal brains are greater than the mean value (1.2 fold) displayed by other sexually dimorphic genes as reported in the adult brain [30]. Similar sex-dependent differences in PSEN1 gene expression were observed in the hippocampus limitedly to the first post-natal week, while no differences were observed in the mature brain. Kristofikova and colleagues also described a sex-dependent dimorphism during post-natal hippocampal development regarding the action of $\mathrm{A} \beta$ peptides on choline carriers in the rat brain, suggesting a role for the $\mathrm{A} \beta$ in the postnatal sexual differentiation of the hippocampal cholinergic system [31]. With regard to the quantitative expression profile of PSENs during post-natal life, we find a relatively high expression of PSEN1 transcripts at P0 in cerebral cortex and hippocampus in both sexes that steadily decreased during the period of synaptogenesis in early post-natal development. Similarly, the levels of nicastrin were high at the early stages of post-natal development and gradually declined to acquire the typical profile of adult brain [32]. The progressive decrease of PSEN1 expression was also observed in the male cerebellum, but not significantly detectable in the female cerebellum. The expression profile of PSEN2 was similar to PSEN1, but our quantitative analysis of PSEN2 transcripts revealed no sex-dependent differences. These data reiterate that expression of the presenilin homologs is 
differentially regulated by sex during brain maturation, suggesting a possible specific functional role exerted by each protein in the development of brain dimorphism. Our findings on the expression of PSEN1 and PSEN2 expression and localization in a specific subsets of neurons are in agreement with previous reports that were mostly focused on PSEN1 [9,32]. However, as far as we know, the issue of if/how sex-influences the expression of presenilins at early post-natal stages of brain development was poorly investigated so far. Observations of this study now indicate that differences between males and females entail both protein levels and localization of PSENs. As for the cerebral cortex, our data show a consistent lack of PSENs expression in layer V pyramidal cells of male rats. This is noteworthy in light of previous observations describing the existence of a different pattern of dendritic arborization of neurons of this layer in young adult rats [33]. Striking differences in PSEN1 and PSEN2 expression between the two sexes were also observed in the cerebellum. In the developing cerebellum neurons acquire fully mature physiological characteristics at three/four weeks of age; this maturation is accompanied by a spatio-temporally regulated series of events, including the migration of granule neurons from the external to the internal granule layer, and the alignment of Purkinje cells in a single row followed by the gradual enlargement of their sophisticated dendritic tree [34]. Our data show that PSEN1 immunostaining is detectable in the IGL of P14 male, but not in female rats; on the other hand Purkinje cells of female rats express a higher level of PSEN1 protein. A similar profile is observed for PSEN2; even detectable earlier (P7) compared to its homologue PSEN1, further strengthening the notion that the functional role(s) played by the two PSENs genes are not redundant. Given that full differentiation and synaptic maturation of both pyramidal neurons of the cerebral cortex and cerebellar Purkinje cells take place over a prolonged period of time, encompassing the first three weeks of post-natal life, it is tempting to hypothesize that sex-related PSENs expression/localization differences in these neurons may contribute to setting the morphologica/functional specificity of the two sexes. In conclusion, the fine-tuning of PSEN1 and PSEN2 expression during post-natal brain development in female and male rats suggests that, besides the $\gamma$-secretase complex, these proteins exert additional functions likely deserving further investigation.

\section{ACKNOWLEDGEMENTS}

The authors would address particular thanks to Dr. Sonal Jhaveri for critical reading of the manuscript. This study was supported by a grant from the Italian Ministry of Health: "Genetic determinants and modulator factors in neurodegenerative diseases: Clinical and experimental model" to A.C and a grant from EC 7th Framework program (FP7/
2007-2013) Grant n. 278486 “Develage” to MT.F.

\section{REFERENCES}

[1] Wolfe, M.S. (2006) The gamma-secretase complex: Membrane-embedded proteolytic ensemble. Biochemistry, 45, 7931-7939. http://dx.doi.org/10.1021/bi060799c

[2] Hutton, M. and Hardy, J. (1997) The presenilins and Alzheimer's disease. Human Molecular Genetics, 6, 16391646. http://dx.doi.org/10.1093/hmg/6.10.1639

[3] Selkoe, D. and Kopan, R. (2003) Notch and presenilin: Regulated intramembrane proteolysis links development and degeneration. Annual Review of Neuroscience, 26, 565-597.

http://dx.doi.org/10.1146/annurev.neuro.26.041002.13133 $\underline{4}$

[4] Berezovska, O., Xia, M.Q., Page, K., Wasco, W., Tanzi, R.E. and Hyman, B.T. (1997) Developmental regulation of presenilin mRNA expression parallels notch expression. Journal of Neuropathology \& Experimental Neurology, 56, 40-44. http://dx.doi.org/10.1097/00005072-199701000-00004

[5] Song, W., Nadeau, P., Yuan, M., Yang, X., Shen, J. and Yankner, B.A. (1999) Proteolytic release and nuclear translocation of notch-1 are induced by presenilin 1 and impaired by pathogenic presenilin 1 mutations. Proceedings of the National Academy of Sciences of the United States of America, 96, 6959-6963. http://dx.doi.org/10.1073/pnas.96.12.6959

[6] Shen, J., Branson, R.T., Chen, D.F., Xia, W., Selkoe, D.J. and Tonegawa, S. (1997) Skeletal and CNS deficits in presenilin 1-deficient mice. Cell, 89, 629-639. http://dx.doi.org/10.1016/S0092-8674(00)80244-5

[7] Conlon, R.A., Reaume, A.G. and Rossant, J. (1995) Notch1 is required for the coordinate segmentation of somites. Development, 121, 1533-1545.

[8] Lee, M.K., Slunt, H.H., Martin, L.J., Thinakaran, G., Kim, G., Gandy, S.E., Seeger, M., Koo, E., Price, D.L. and Sisodia S. (1996) Expression of presenilin 1 and 2 (PS1 and PS2) in human and murine tissues. The Journal of Neuroscience, 16, 7513-7525.

[9] Moreno-Flores, M.T., Medina, M. and Wandosell, F. (1999) Expression of presenilin 1 in nervous system during rat development. Journal of Comparative Neurology, 410, 556-570.

http://dx.doi.org/10.1002/(SICI)1096-9861(19990809)41 0:4<556::AID-CNE4>3.0.CO;2-Q

[10] Kumar, A. and Thakur, M.K. (2012) Presenilin 1 and 2 are expressed differentially in the cerebral cortex of mice during development. Neurochemistry International, 61, 778-782. http://dx.doi.org/10.1016/j.neuint.2012.07.001

[11] Kang, D.E., Yoon, I.S., Repetto, E., Busse, T., Yermian, N., Ie, L. and Koo, E.H. (2005) Presenilins mediate phosphatidylinositol 3-kinase/AKT and ERK activation via select signaling receptors. Selectivity of PS2 in platelet-derived growth factor signaling. The Journal of Biological Chemistry, 280, 31537-31547.

http://dx.doi.org/10.1074/jbc.M500833200 
[12] Satoh, J. and Kuroda, Y. (1999) Constitutive and cytokine-regulated expression of presenilin-1 and presenilin-2 genes in human neural cell lines. Neuropathology and Applied Neurobiology, 25, 492-503.

http://dx.doi.org/10.1046/j.1365-2990.1999.00209.x

[13] Ghosh, S. and Thakur, M.K. (2008) PS1 expression is downregulated by gonadal steroids in adult mouse brain. Neurochemical Research, 33, 365-369. http://dx.doi.org/10.1007/s11064-007-9424-8

[14] Ghosh, S. and Thakur, M.K. (2008) PS2 protein expression is upregulated by sex steroids in the cerebral cortex of aging mice. Neurochemistry International, 52, 363367. http://dx.doi.org/10.1016/j.neuint.2007.07.015

[15] Bowen, R.L., Verdile, G., Liu, T., Parlow, A.F., Perry, G., Smith, M.A., Martins, R.N. and Atwood, C.S. (2004) Luteinizing hormone, a reproductive regulator that modulates the processing of amyloid-beta precursor protein and amyloid-beta deposition. The Journal of Biological Chemistry, 279, 20539-20545. http://dx.doi.org/10.1074/jbc.M311993200

[16] Placanica, L., Zhu, L. and Li, Y.M. (2009) Gender- and age-dependent gamma-secretase activity in mouse brain and its implication in sporadic Alzheimer disease. PLoS One, 4, e5088. http://dx.doi.org/10.1371/journal.pone.0005088

[17] Gallagher, J.J., Minogue, A.M. and Lynch, M.A. (2013) Impaired performance of female APP/PS1 mice in the Morris water maze is coupled with increased $\mathrm{A} \beta$ accumulation and microglial activation. Neurodegenerative Diseases, 11, 33-41. http://dx.doi.org/10.1159/000337458

[18] Thakur, M.K. and Ghosh, S. (2007) Age and sex dependent alteration in presenilin expression in mouse cerebral cortex. Cellular and Molecular Neurobiology, 27, 1059-1067. http://dx.doi.org/10.1007/s10571-007-9214-5

[19] European Economic Community (1986) European Council Directive 86/609/EEC on the approximation of laws, regulations and administrative provisions of the member states regarding the protection of animals used for experimental and other scientific.

http://eurlex.europa.eu/LexUriServ/LexUriServ.do?uri=C ELEX:31986L0609:EN:HTM

[20] Piscopo, P., Bernardo, A., Calamandrei, G., Venerosi, A., Valanzano, A., Bianchi, D., Confaloni, A. and Minghetti, L. (2008a) Altered expression of cyclooxygenase-2, presenilins and oxygen radical scavenging enzymes in a rat model of global perinatal asphyxia. Experimental $\mathrm{Neu}$ rology, 209, 192-198.

http://dx.doi.org/10.1007/s10571-007-9214-5

[21] Piscopo, P., Marcon, G., Piras, M.R, Crestini, A, Malvezzi Campeggi L., Deiana E., Cherchi R., Tanda F., Deplano A., Vanacore N., Tagliavini F., Pocchiari M., Giaccone G. and Confaloni A.. (2008b) A novel PSEN2 mutation associated with a peculiar phenotype. Neurology, 70, 1549-1554.

http://dx.doi.org/10.1212/01.wnl.0000310643.53587.87

[22] Piscopo, P., Talarico, G., Crestini, A., Gasparini, M., Malvezzi-Campeggi, L., Piacentini, E., Lenzi, G. L., Bruno, G. and Confaloni, A. (2010) A novel mutation in the predicted TM3 domain of the PSEN2 gene in an Italian pedigree with a typical Alzheimer's disease. Journal of Alzheimer's Disease, 20, 43-47.

[23] Andersen, K., Launer, L.J., Dewey M.E., Letenneur, L., Ott, A., Copeland, J.R., Dartigues, J.F., Kragh-Sorensen, P, Baldereschi, M., Brayne, C., Lobo, A., Martinez-Lage, J.M., Stijnen, T., Hofman, A. and EURODEM Incidence Research Group (1999) Gender differences in the incidence of $\mathrm{AD}$ and vascular dementia: The EURODEM studies. Neurology, 53, 1992-1997.

http://dx.doi.org/10.1212/WNL.53.9.1992

[24] Fratiglioni, L., Viitanen, M., von Strauss, E., Tontodonati, V., Herlitz, A. and Winblad, B. (1997). Very old women at highest risk of dementia and Alzheimer's disease: Incidence data from the Kungsholmen Project, Stockholm. Neurology, 48, 132-138. http://dx.doi.org/10.1212/WNL.48.1.132

[25] Mani, S.T. and Thakur M.K. (2006) In the cerebral cortex of female and male mice, amyloid precursor protein (APP) promoter methylation is higher in females and differentially regulated by sex steroids. Brain Research, 1067, 43-47. http://dx.doi.org/10.1016/j.brainres.2005.10.006

[26] Drummond, E.S., Martins, R.N., Handelsman, D.J. and Harvey, A.R. (2012) Altered expression of Alzheimer's disease-related proteins in male hypogonadal mice. Endocrinology, 153, 2789-2799. http://dx.doi.org/10.1210/en.2011-2003

[27] Cooke, G.M., Price, C.A. and Oko, R.J. (1998) Effects of in utero and lactational exposure to 2,3,7,8-tetrachlorodibenzo-p-dioxin (TCDD) on serum androgens and steroidogenic enzyme activities in the male rat reproductive tract. The Journal of Steroid Biochemistry and Molecular Biology, 67, 347-54. http://dx.doi.org/10.1016/S0960-0760(98)00127-7

[28] Metzger, E., Wissmann, M., Yin, N., Müller, J.M., Schneider, R., Peters, A.H., Günther, T., Buettner, R. and Schüle, R. (2005) LSD1 demethylates repressive histone marks to promote androgen-receptor-dependent transcription. Nature, 437, 436-439.

[29] Xu, J., Deng, X., Watkins, R. and Disteche, C.M. (2008) Sex-specific differences in expression of histone demethylases Utx and Uty in mouse brain and neurons. The Journal of Neuroscience, 28, 4521-4527. http://dx.doi.org/10.1523/JNEUROSCI.5382-07.2008

[30] Yang, X., Schadt, E.E., Wang, S., Wang, H., Arnold, A.P., Ingram-Drake, L., Drake, T.A. and Lusis, A.J. (2006) Tissue-specific expression and regulation of sexually dimorphic genes in mice. Genome Research, 16, 995-1004. http://dx.doi.org/10.1101/gr.5217506

[31] Kristofiková, Z., Rícny, J., Kozmiková, I., Rípová, D., Zach, P. and Klaschka, J. (2006) Sex-dependent actions of amyloid beta peptides on hippocampal choline carriers of postnatal rats. Neurochemical Research, 31, 351-360.

[32] Kodam, A., Vetrivel, K.S., Thinakaran, G. and Kar, S. (2008). Cellular distribution of gamma-secretase subunit nicastrin in the developing and adult rat brains. Neurobiology of Aging, 29, 724-738.

http://dx.doi.org/10.1016/j.neurobiolaging.2006.12.005

[33] Kolb, B. and Stewart, J. (1991) Sex-related differences in dendritic branching of cells in the prefrontal cortex of rats. 
Journal of Neuroendocrinology, 3, 95-99. http://dx.doi.org/10.1111/j.1365-2826.1991.tb00245.x

[34] Sotelo, C. (2004) Cellular and genetic regulation of the development of the cerebellar system. Progress in Neurobiology, 72, 295-339.

http://dx.doi.org/10.1016/j.pneurobio.2004.03.004 